\title{
Oralité et figuration de la mémoire chez Césaire, Chamoiseau et Monénembo
}

\author{
Elizabeth Catherine Saint, Université de Victoria, Canada
}

Alors que l'oral est l'expression naturelle, mouvante et non linéaire de la pensée, l'écrit en est sa forme artificielle, construite et organisée. Typiquement, quand l'écrit intègre l'oral, il le représente sous forme de discours direct qui simule la production orale puisqu'en passant à l'écrit, cette dernière prend une forme fixe et linéaire qui ne lui est plus naturelle. L'oralité, qui est tout ce qui «se rattache à une production collective orale, c'est-à-dire à des formes traditionnelles et populaires comme le conte, l'énigme, la devinette, le proverbe, [...] les chants » (Pestre de Almeida, «Ariettes retrouvées », 272), est elle-même simulée quand elle vient à être représentée à l'écrit. L'écrivain se voit amené à intégrer une forte thématique de la parole et à recourir à une variété de stratégies narratives ou stylistiques. D’une part, les récits traditionnels oraux composeront la forme du texte qui deviendra, par exemple, chant ou conte, ou alors ils seront insérés en intertextes. D'autre part, la narration reproduira, par la création littéraire et stylistique, les caractéristiques de l'oral, par exemple son rythme, sa non-linéarité, son lexique, etc.

«Puisque l'écriture est un outil de séduction » (Descas 44), les marques de l'oralité ne sont pas gratuitement imposées au texte et elles jouent un rôle précis que nous tenterons de déterminer ici à l'intérieur des trois œuvres suivantes : Cahier d'un retour au pays natal d'Aimé Césaire, Chronique des sept misères de Patrick Chamoiseau, et L'Aîné des orphelins de Tierno Monénembo. Pour ce faire, nous relèverons tout d'abord les stratégies auxquelles les auteurs ont fait appel pour représenter l'oralité dans leurs œuvres respectives. Puis nous examinerons le rôle que joue l'oralité dans chacune des œuvres. Notre analyse conclura que l'oralité, tout en prenant des formes diverses chez Césaire, Chamoiseau et Monénembo, joue un rôle commun dans les trois œuvres : celui de figuration de la mémoire.

De par son usage d'un français très académique à l'écrit et du fait qu'il a toujours choisi de s'exprimer en français à ses compatriotes lors de ses interventions publiques, Aimé Césaire a été critiqué par bon nombre d'intellectuels antillais qui ont conclu, assez rapidement, que le poète martiniquais avait «un problème mental avec sa langue, son identité d'enfance, sa culture, 
son amour pour le pays natal »(Prudent 31). Ainsi, le Cahier d'un retour au pays natal, dont la lecture a souvent été considérée hermétique, fait appel à un niveau de français supérieur et est une démonstration de la connaissance remarquable qu'avait Césaire de la langue du colonisateur. André Breton mentionne d'ailleurs dans sa préface à l'édition de 1947 que Césaire est « un Noir qui manie la langue française comme il n'est pas aujourd'hui un Blanc pour la manier » (Césaire 80). Cependant, à y regarder de plus près, il est possible de constater que, malgré la prépondérance du français, le texte du Cahier est métissé et qu'il contient des traces d'autres langues et d'autres cultures, dont le créole. Lilian Pestre de Almeida relève une diglossie antillaise dans l'expression «au bout du petit matin » qui serait l'équivalent de l'expression créole « au pipiri du jour» (Aimé Césaire, 128). Nous notons, pour notre part, d'autres traces d'une diglossie français/créole dans les syntagmes composés renvoyant au rythme du créole « de-peur-que-ça-ne-suffise-pas » (15) (possible équivalent de «pou si an ka pa ni assé ») ou dans la répétition à fonction amplificatrice typique du créole « les cloches... la pluie... qui tintent, tintent, tintent... » (17). Les deux vers suivants sont un autre exemple de la répétition à valeur d'amplification et de la volonté de récréer le rythme de la langue créole à l'aide du syntagme composé : « les nègres-sont-tous-les-mêmes, je-vous-le-dis / les vices-tous-les-vices, c'est-moiqui-vous-le-dis » (Césaire 35).

En fait, le poème de Césaire est bien plus que diglossique, il est polyglossique. LambertFélix Prudent rappelle à ce propos les travaux d'Annie Dyck en 1988', et de René Hénane, en $2004^{2}$. Dyck avait entrepris de relever tous les mots «étrangers» du Cahier afin de montrer le substrat créole contenu dans le poème : «L'hypothèse polylectale la conduit à identifier les langues anciennes (le grec et le latin très fréquents, le français médiéval, les emprunts à diverses langues d'Afrique et d'Amérique latine, l'espagnol, le portugais et surtout des “traces créoles") » (Prudent 38). Parmi les noms antillais qu'elle relève, tels que morne et ravet, elle note aussi la présence de noms moins culturellement connotés mais qui sont utilisés à l'antillaise, tels que savane et vieux. Avec son Glossaire des termes rares, Hénane a, lui, permis d'identifier « les termes savants qui désignent des référents antillais » et inversement. Cela se retrouve dans l'exemple des Cayes (Césaire 26) qui signifient à la fois « des rochers de vase ou de corail » et « des cases ou maisons antillaises ». Ces traces diglossiques avec le créole confirment que Césaire fait « un usage poétiquement créole des ressources du français » (Depestre 165). 
L'oralité de Césaire se dégage aussi dans les transformations que subit le texte à chacune de ses nombreuses éditions. Tel «l'héritier du griot ou du conteur populaire » (Pestre de Almeida, «Ariettes Retrouvées », 272), Césaire livre un poème en mouvement constant, dont la version finale est incertaine et qui s'inscrit plus dans l'oral que l'écrit: «L'une des contradictions fondatrices du poème est l'opposition lettre morte vs parole vivante [...], ce texte mouvant, l'est, parce qu'en profondeur, pour l'auteur, il se rattache à la parole et non pas à la lettre » (Pestre de Almeida, Aimé Césaire, 127).

Pour finir, l'oralité est renforcée, dans le poème de Césaire, par l'usage de «genres mnémoniques » typiques des récits issus des sociétés à tradition orale et ayant pour but de transmettre «les informations intergénérationnelles les plus pertinentes pour la survie de la communauté » (Bernabé 52), tels les dictons, « rappelez-vous-le-vieux-dicton / battre-un-nègre, c'est le nourrir » (Césaire 35), mais aussi les contes et les chants magico-religieux :

Grandvorka - celui-là je sais seulement qu'il est mort, broyé par un soir de récolte, c'était paraît-il son travail de jeter du sable sous les roues de la locomotive en marche, pour lui permettre, aux mauvais endroits, d'avancer. (Césaire 54)

voum rooh oh

à charmer les serpents à conjurer les morts voum rooh oh

à contraindre la pluie à contrarier les raz de marée voum rooh oh à empêcher que ne tourne l'ombre voum rooh oh que mes cieux à moi s'ouvrent (Césaire 30)

La faculté humaine essentielle à toute culture orale est la mémoire, moyen premier de retenir ce qui est dit oralement et d'en garantir sa transmission d'une génération à l'autre. Or, « la mémoire ne se fait pas sans répétitions, sans ressassement 》 (Glissant, «Le chaos-monde », 112) et le Cahier, parce qu'il mêle le texte à réciter de par son genre poétique - le texte à mémoriser de par ses répétitions quasi-litaniques -, est un texte fondamentalement oral.

L'histoire de Pipi et du marché de Fort-de-France, ainsi que les multiples autres anecdotes de Chronique des sept misères nous sont racontées par un djobeur dont le lecteur ne connaîtra jamais la réelle identité, même s'il sait qu'il est membre du groupe des cinq maîtres djobeurs du 
marché (75). Ce narrateur est tantôt un nous homodiégétique, protagoniste, tantôt un il hétérodiégétique, observateur extérieur. Il joue ainsi le rôle d'un narrateur qui, bien que subjectivement impliqué dans ce récit, nous le narre de la manière la plus objective possible. Cette incertitude narrative permet certainement d'ajouter un côté véridique aux histoires racontées, en respect de la chronique ${ }^{3}$, mais aussi de ne pas faire du narrateur la seule voix du roman. C'est ainsi que des narrateurs seconds interviennent à leur bon gré au moyen du discours indirect libre, tels Man Goul racontant 1'histoire d'Anastase (99-117) ou Pipi racontant, tant au lecteur qu'à Clarine elle-même, l'histoire de la vie de cette dernière (57-62). Ces effets stylistiques donnent au roman sa tonalité : nous sommes en présence d'un conte pris en charge par une multitude de conteurs s'adressant eux-mêmes à une multitude de «messieurs et dames de la compagnie »(Chamoiseau, Chronique, 15), public représentatif de celui auquel les conteurs s'adressaient lors de veillées nocturnes et qui est au cœur de la poétique de l'oralité des Antilles (Chamoiseau, «Que faire de la parole ? », 157). En effet, la nuit est « le lieu de la parole créole. [...] C'est une fois la nuit tombée qu'on raconte la vie des ancêtres aux enfants, et c'est là encore qu'on fête leur mort au cours des veillées » (Ludwig 18). Ainsi, en manipulant les formes narratives, Chamoiseau reproduit le contexte de la parole de nuit et son roman est proche de la performance orale retranscrite.

L'autre marque de l'oralité chez Chamoiseau provient de son usage du créole qui « est le véhicule originel $[\mathrm{du}]$ moi profond, de [1']inconscient collectif, [du] génie populaire » des Antillais (Bernabé, Confiant et Chamoiseau 43). On trouvera le créole en italique ou non, traduit partiellement ou entièrement, à l'occasion des rares interactions au discours direct entre les personnages, ou à l'intérieur de la narration sous forme d'interjections et d'onomatopées reflétant le rythme de la parole orale créole (hak, flap, mi, fout, glouque, etc.), sous forme de commentaires à valeur émotive ou phatique (apa kouyannad, pou si anka, tonnan di sô, mésiézé-dames, é kri é kra, sacrée manman de la rumeur, etc.), ou sous forme de proverbes antillais (ich tig paka fêt san zong). Toutefois, comme le remarque Marie-Josèphe Descas dans sa thèse de 1995, la langue du roman reste essentiellement française. Elle précise même que « si l'on considère la faible présence du créole on est [...] tenté de parler d'ornements linguistiques, d'artifices visant à créer un effet d'oralité » (95). Ainsi, pour parvenir à faire « un usage fécond de l'interlecte » qui « constitu[e] la voie d'accès à un ordre de réalité susceptible de conserver à 
[la] créolité sa complexité fondamentale, son champ référentiel difracté » (Bernabé, Confiant et Chamoiseau 49-50), une subtile créolisation de la langue française s'opère sous la plume de Chamoiseau, au point qu'il est difficile pour le lecteur peu attentif de réellement prendre conscience qu'il est face à une nouvelle langue. Le français s'anime et son goût de l'abstrait fait place au concret de la réalité créole. Ainsi, l'on trouvera des calques d'expressions créoles tels que «il lui dit deux mots, quatre paroles»(Chamoiseau, Chronique, 122) ou «n'osa pièce temps » (138). Certaines expressions françaises prendront une tournure plus concrète, comme «Elle allait saisir [...] le destin par un bout différent »(32), «Parole n'ayant pas d'épaules » (145), « quand le soleil ramasse ses dernières affaires » (174) ou encore « les petits producteurs [...] se voyaient coiffer par le peigne de la dèche » (199). D’autres expressions se réfèreront à des réalités antillaises, telles la faune - «plus pensif qu'un zandoli devant une mouche » (142) -, la flore - «nous avions depuis longtemps chuté du calebassier de l'existence »-(221), la mer « les quatre fers à l'embellie » (206). Comme le dit Édouard Glissant dans la préface de Chronique des sept misères, Chamoiseau «marche à cette lisière de l'oral et de l'écrit où se joue une des perspectives actuelles de la littérature » («Un marqueur de paroles », 6).

Finalement, la chronique, en ce qu'elle est porteuse d'une histoire réelle ou fictive et qu'elle contribue à alimenter la mémoire collective, est aussi une marque de l'oralité du roman. Le récit est « recueil de faits historiques » et nous fournit des informations sur l'histoire de la Martinique tels que les évènements de la Seconde Guerre mondiale aux Antilles, l'histoire des esclaves africains via les paroles rêvées d'Afoukal ou encore la départementalisation de l'île. Il est aussi «récit de faits sociaux authentiques » qui, à travers ses personnages, si pauvres, si inutiles, si vulgaires, si inaptes soient-ils (Bernabé, Confiant et Chamoiseau 40), nous donnent à voir la vie martiniquaise, sa misère, sa famille, son marché, sa religion, etc. Finalement, il est «ensemble de commérages » et abonde en rumeurs et en paroles diverses qui apportent une dimension merveilleuse au roman et révèlent les nombreuses superstitions et croyances antillaises. Circulant entre 1'Histoire et les histoires, l'écriture de Chamoiseau « donn[e] à voir les héros insignifiants, les héros anonymes, les oubliés de la Chronique coloniale » (40) et les grave dans la mémoire du peuple antillais à l'aide d'une littérature qui se situe à mi-chemin entre l'oral et l'écriture, dans ce que l'auteur lui-même appelle l'oraliture, soit « une production orale qui se distingu[e] de la parole ordinaire par sa dimension esthétique » (Chamoiseau, «Que faire 
de la parole ?»,153).

Écrit dans le cadre du projet Rwanda : écrire par devoir de mémoire ${ }^{4}$, le roman de Tierno Monénembo assume l'une des trois formes de narration principales du génocide ${ }^{5}$, à savoir «le rôle donné aux personnages-témoins dans les œuvres littéraires d'écrivains extérieurs aux événements »(Germanotta 8). L'Aîné des orphelins est le témoignage d'un jeune garçon, Faustin Nsenghimana, rescapé du massacre des Tutsi partiellement amnésique. Les marques de l'oralité du récit révèlent l'immaturité du narrateur et se situent au niveau de son énonciation incertaine, voire enfantine (avènements, pédrophile, taumatrismes, busenessmen, le Ouatican, les NotionsUnies). De plus, l'on trouve des marqueurs du discours direct, tels les points d'exclamation ou d'interrogation, ainsi que des reproductions manuscrites des caractéristiques phonétiques de l'oral, comme «Y avait pas lit plus moelleux ! (Monénembo 31), qui sont typiques du discours indirect libre et qui, ajoutées à l'utilisation de la citation libre dans la narration, permettent de reproduire le rythme non-linéaire de la parole et de la pensée sans créer de rupture dans la trame narrative :

Le travail aussi, ma foi, ne se passait pas toujours sans incident. Par exemple, ce jour où un client m'accusa d'avoir volé son autoradio. Ce crétin de Canisius ! Combien de fois lui avais-je dit de ne pas toucher aux autoradios et aux bijoux : rien que du pratique, du discret, de l'utilitaire (de l'argent, des vêtements, de la nourriture, à la rigueur des calculettes ou des appareils photo, ça oui !). Le bougre, il me saisit par les deux oreilles et me leva, mes pieds à la hauteur de sa poitrine. (38)

Ces effets d'oralité humanisent Faustin, le détachent de son rôle de narrateur et nous donnent à voir un personnage libre de s'exprimer comme il l'entend, que cela nous interroge, nous indigne, nous choque, nous apitoie ou nous fasse sourire. Faustin devient, par l'oralité du texte, le témoin par excellence : celui qui raconte ce qu'il a vécu, avec toutes les émotions que cela comporte.

Le flou temporel du récit, créé par un amalgame d'analepses qui déroutent le lecteur et le forcent à reconstituer la chronologie des évènements, contribue aussi à l'oralité du récit. En effet, il reproduit le processus non-linéaire de reconstruction du récit de vie, à l'image de la mémoire morcelée de Faustin, puisque «L'exactitude de l'information livrée par l'enquête orale ne l'est que par à-coups, de manière fragmentée, détournée et sédimentaire; [...] elle ne s'offre 
normalement pas d'une seule volée, mais se construit par strates » (Niang 132). Néanmoins, il est possible que cette désorganisation temporelle provienne du fait qu'une partie des souvenirs du jeune Faustin découlent du récit des autres, comme il l'indique en précisant : «mes souvenirs du génocide s'arrêtent là. Le reste, on me l'a raconté par la suite » (Monénembo 124). Le témoignage devient alors polyphonique, témoignage d'une collectivité, expression d'une multitude de voix. C'est ainsi que la mort de l'Italienne et la fuite des Tutsi sont contées par le sorcier Funga (4-6) et qu'une conversation entre un jeune soldat du Front patriotique rwandais et son capitaine nous apprend la présence et l'action des milices armées, FAR et PPR $^{6}$ (25). De même, c'est lors d'un dialogue entre Musinkôro et un autre orphelin que l'on apprend la présence des ONG à Kigali (35), et c'est Hitimana, un enfant de l'orphelinat, qui raconte le déroulement des retrouvailles de Faustin avec ses sœurs et son petit frère (50-52). Puis, il est dit que c'est un adulte «lassé par les nécessités de l'oubli [qui] évoquât ce qui s'était passé avant : la saignée de 1959, celle de 1964, celle de 1972, etc. » (92), et finalement, c'est une femme qui décrit à Faustin ce qui s'est passé alors qu'elle vient de le découvrir au milieu des cadavres après le massacre (124). Faustin retrouve la mémoire à partir d'« une pluralité de points de vue et de voix narratives, laissant percevoir à quel point la réalité du génocide est instable, incertaine et à quel degré elle ne cesse de se dérober » (Semujanga, «Le Génocide », 115). Le témoin de cette histoire devient le peuple rwandais, tant celui qui a survécu au génocide que celui qui a disparu.

Pour finir, la littérature africaine traditionnelle est fondamentalement orale et elle « permet la diffusion du savoir, de la culture, des coutumes, des usages, de la pensée et de la philosophie africaines à l'aide d'une rhétorique essentiellement centrée sur la mémoire » (Saganogo 488, notre traduction). Pour cela, elle recourt à divers genres parmi lesquels se trouvent la chanson, la poésie, le théâtre, mais aussi les proverbes, les légendes et les fables. Le roman de Monénembo contient de nombreuses traces de cette oralité traditionnelle africaine particulièrement visibles dans les proverbes, «Les morts ne souffrent pas, ils se reposent d'avoir vécu » (25), « Le monde, il marche, même si c'est souvent de travers » (33), «Celui-là va mourir, qui croit se passer des autres » (42), «Si tu hais un homme, laisse-le vivre!»(111). Ces proverbes sont souvent employés pour tenter de comprendre l'incompréhensible de la situation, de même que le rocher de la Kagera qui fait référence à la naissance du peuple rwandais, tente d'expliquer la raison du génocide. Le rocher de Kinani, dans la légende originale, «servait de lieu où se rencontraient les 
forces antagonistes, celles du ciel, de la terre habitée et du monde souterrain. En le déplaçant, on a détruit l'équilibre entre ces trois forces et conduit au chaos actuel» (Semujanga, «Les Méandres », 109). Ajoutés à cette légende et aux nombreux proverbes, se trouvent les dictons et fables à fonction d'apprentissage de la vie pour les plus jeunes, tel « La barbe n'est pas tout, non ! S’il en était ainsi, le bouc serait le plus sage du village » (Monénembo, 23) ou encore :

La fable de mon vaurien de Théoneste de père est encore toute fraîche dans ma tête : «Mensonge et Vérité sont les premiers habitants de la terre. Vérité est le frère aîné mais comme Mensonge est le plus doué, eh bien, c'est lui qui mène le monde. N'oublie jamais ça, petit ! » (79)

D'une part, ces intertextes - proverbes, fables, dictons, légendes - affirment la volonté de Monénembo de recourir à l'oralité africaine dans sa création littéraire. D'autre part, ils convoquent la voix de ceux qui ont disparu, comme celle du sorcier parti en exil et celle des parents de Faustin qui ont péri dans le génocide.

Comme l'Histoire de nombreux pays d'Afrique et des Antilles « est naufragée dans l'Histoire coloniale », il est urgent de préserver la mémoire populaire (Bernabé, Confiant et Chamoiseau 36-37). Dans ces cultures traditionnellement orales, l'on s'interroge sur la capacité de l'écriture à y contribuer : «D'autres peuples se servent de l'écriture pour fixer le passé ; mais cette invention a tué la mémoire chez eux ; ils ne sentent plus le passé car l'écriture n'a pas la chaleur de la voix humaine » (Djeli Mamadou Kouyaté, cité dans Diakité 57). C'est cette chaleur de la voix humaine que l'oralité apporte à la littérature d'Afrique et des Antilles. Cela passe par la mise en place de diverses stratégies stylistiques, esthétiques, linguistiques, etc. qui tentent toutes de rendre compte de la nature orale traditionnelle de ces sociétés et de l'importance de la transmission intergénérationnelle de leur culture, de leur savoir, de leur Histoire et de leurs histoires.

Ainsi, quoique Césaire écrive en français et fasse abstraction du créole, il semble hâtif de conclure que, par un suicide esthétique, le poète a tué la densité orale et l'authentique créolité de son texte (Bernabé, Confiant et Chamoiseau 45). Par un métissage de la langue française qui s'effectue subtilement, la créolité césairienne se diffuse dans les pages du Cahier presqu'à l'insu de tous. En déstabilisant la syntaxe pure du français, en aliénant son lexique établi, il déconstruit l'identité même du colonisateur et par là renforce le message de gloire et d'indépendance 
antillaises qu'il désire transmettre aux générations futures. Bref, Césaire montre ici combien, lui aussi, il «l'a conquise, cette langue française » (Bernabé, Confiant et Chamoiseau 46).

De son côté, c'est à l'aide de la multiplicité des voix narratives et de la pluralité des identités du conteur de cette chronique que Chamoiseau restitue une portion de l'histoire martiniquaise et donne voix à ceux que l'Histoire a si souvent réduits au silence. Sous sa plume, la langue française se voit métamorphosée afin de mieux rendre compte de ce que sont les Antilles : «le lieu de contact et de confrontation entre le monde européen de l'écrit, de l'alphabétisation et des traditions littéraires d'une part, et le monde de l'oralité, de la langue créole, du conteur, de la fête populaire de l'autre » (Ludwig 15).

Dans L'Aîné des orphelins, la part d'oralité est, elle, ancrée dans le désir qu'a le roman d'agir comme témoignage du génocide rwandais. Cela a lieu à travers le récit de vie de Faustin avec tout ce qu'il contient de contradictoire, de déstructuré, d'elliptique et de refoulé. Avec l'intégration de légendes rappelant l'origine du Rwanda, de proverbes expliquant la philosophie du peuple rwandais, et de dictons et fables permettant la transmission du savoir rwandais d'une génération à l'autre, le roman acquiert un rôle proche de celui du griot. Ce griot aux voix multiples est « le gardien de la mémoire collective » (Kemedjio 30) et il transmet l'histoire d'un peuple. Le roman devient alors porteur du patrimoine rwandais et témoigne d'une culture que le génocide a atrophiée. Il se veut mémoire du peuple pour le peuple.

Pour conclure, des procédés mnémoniques mis en place dans la structure du poème de Césaire, au témoignage polyvocal de Monénembo, en passant par les rumeurs et légendes de Chamoiseau, ces œuvres sont toutes porteuses d'un message qu'elles cherchent à inscrire par le dire dans la mémoire collective.

\section{Ouvrages cités}

Bernabé, Jean. « De l'oralité à la littérature antillaise : figures de l'Un et de l'Autre ». Littérature et dialogue interculturel : culture française d'Amérique. Dir. Françoise Tétu de Labsade. Sainte-Foy : PU Laval, 1997.

---, Raphaël Confiant et Patrick Chamoiseau. Éloge de la créolité. Ed. bilingue français / anglais. Paris : Gallimard, 1993.

Césaire, Aimé. Cahier d'un retour au pays natal. Paris : Présence africaine, 1983. 
Chamoiseau, Patrick. Chronique des sept misères. Paris : Gallimard, 2006.

---. «Que faire de la parole ?» Écrire la parole de nuit: la nouvelle littérature antillaise, nouvelles, poèmes et réflexions poétiques. Dir. Ralph Ludwig. Paris : Gallimard, 1994. $151-158$.

«Chronique ». Le Grand Robert de la langue française. 2011. Version électronique 2.2. 29 janvier 2013 <http://gr.bvdep.com.ezproxy.library.uvic.ca/version-1/gr.asp>.

Depestre, René. «Les aventures de la créolité ». Écrire la parole de nuit : la nouvelle littérature antillaise, nouvelles, poèmes et réflexions poétiques. Dir. Ralph Ludwig. Paris: Gallimard, 1994. 159-170.

Descas, Marie-Josèphe. «Oralité écrite et créolité romanesque ». Thèse. U of Pennsylvania, 1995.

Diakité, Boubakary. «Entre désécriture et oralité feinte: En attendant le Vote des bêtes sauvages ou la rhétorique de l'instabilité ». Nouvelles Études Francophones 25.2 (2010) : $55-71$.

Germanotta, Maria Angela. «L'écriture de l'inaudible. Les narrations littéraires du génocide au Rwanda ». InterFrancophonies (2010) : 1-34.

Glissant, Édouard. «Un marqueur de paroles (préface)». Chronique des sept misères. Patrick Chamoiseau. Paris : Gallimard, 2006. 3-6.

---. «Le chaos-monde, l'oral et l'écrit». Écrire la parole de nuit: la nouvelle littérature antillaise, nouvelles, poèmes et réflexions poétiques. Dir. Ralph Ludwig. Paris: Gallimard, 1994. 111-129.

Ludwig, Ralph. «Écrire la parole de nuit». Écrire la parole de nuit : la nouvelle littérature antillaise, nouvelles, poèmes et réflexions poétiques. Dir. Ralph Ludwig. Paris: Gallimard, 1994. 13-25.

Monénembo, Tierno. L'Aîné des orphelins. Paris : Seuil. 2000.

Niang, Sada. «Particularités orales des récits de vie dans Le quatrième siècle et Tout-Monde d'Édouart Glissant ». Récits de vie de l'Afrique et des Antilles : enracinement, errance, exil. Dir. Suzanne Crosta. Sainte-Foy, Québec : GRELCA, 1998. 129-141.

Pestre de Almeida, Lilian. Aimé Césaire : Cahier d'un retour au pays natal. Paris : Harmattan, 2008. 
---. «Ariettes retrouvées, contes recréés : Quelques aspects de la création chez Césaire dans ses rapports avec l'oralité ». Meta : Journal des traducteurs 31.3 (1986) : 272-290.

Prudent, Lambert-Félix. «Aimé Césaire : contribution poétique à la construction de la langue martiniquaise ». Aimé Césaire à l'œuvre: Actes du colloque international, Paris, 8-9 octobre 2008. Dir. Marc Cheymol et Philippe Ollé-Laprune. Paris : Archives contemporaines, 2010. 21-45.

Saganogo, Brahiman. «Evolución de la literatura africana de expresión francófona : De la oralidad a la escritura ». Estudios de Asia y Africa 43.2 (136) (2008) : 487-499.

Semujanga, Josias. «Le Génocide des Tutsi dans la fiction narrative ». French Cultural Studies 20.2 (2009) : 111-132.

---. «Les Méandres du récit du génocide dans L'Aîné des orphelins ». Études littéraires 35.1 (2003) : 101-115.

\section{Notes}

1. Mémoire de DEA obtenu à l'Université des Antilles et de la Guyane et intitulé «Le langage césairien, approche d'une écriture polyglossique ».

2. Ouvrage intitulé Glossaire des termes rares dans l'œuvre d'Aimé Césaire et publié aux Éditions J.M. Place. 3. Selon le Grand Robert de la langue française, une chronique est à la fois " un recueil de faits historiques, rapportés dans l'ordre de leur succession », « un récit qui met en scène des personnages réels ou fictifs et évoque des faits sociaux, historiques, authentiques», et « un ensemble des nouvelles qui circulent sur les personnes ».

4. Ce projet a eu lieu entre 1995 et 2000 à l'initiative de l'Association Arts et Médias d'Afrique de Lille et il a invité une dizaine d'auteurs africains francophones non-rwandais à écrire des textes en résidence, sur les lieux du génocide.

5. Les deux autres formes mentionnées par Germanotta sont « les témoignages directs en français rendus en Europe, transcrits et rédigés par des écrivains professionnels » et « les témoignages directs recueillis sur les lieux, traduits du kinyarwanda en français-rwandais et réécrits par l'auteur ».

6. FAR : Forces armées rwandaises ; FPR : Front patriotique rwandais. 